\title{
Bab Tut de la medina de Tetuán (Marruecos): estudio y datos para su conservación
}

Bab Tut of the medina of Tetouan (Morocco): study and data for its conservation

\section{Jaime Vergara-Muñoz a ${ }^{\text {, Miguel Martínez-Monedero }}{ }^{\text {b }}$}

Universidad de Granada, Granada, Spain

a jaimevergara3@gmail.com; ${ }^{\mathrm{b}}$ miguel@mm-arquitectura.com

\begin{abstract}
At the beginning of the Spanish Protectorate in Morocco (1912-1956) the medina of Tetouan, with its walls and gates, was perceived as a fundamental part of the traditional city that was to be conserved. It is interesting to consider, in this sense, the concern that since the war of Tetuan (1859) was to obtain an adequate graphic representation of this architecture. Among the Maps of the Spanish Army Geographical Service are the first drawing that were made (scale 1:100) of the gates of the medina. They are signed by Francisco Gómez Jordana; Alejo Corso and Eduardo Álvarez in 1888. The purpose of this study is to publish the Bab Tut survey and its description. Thanks to this drawing we can know the exact status of the gate before the Spanish occupation and establish a documentary base that facilitates the recovery of this defensive heritage of the city of Tetouan.
\end{abstract}

Keywords: Gates, medina, Tetouan, Morocco.

\section{Introducción}

La medina de Tetuán tiene siete puertas de entrada. No existe una bibliografía específica sobre la evolución histórica de las puertas ni de la muralla. Tampoco está unificada la documentación gráfica, ni la planimétrica. Cada una de las puertas ha recibido numerosas actuaciones restauradoras, más destinadas a la consolidación que a la fiel restauración. De ahí que, con el paso de los años, posean elementos añadidos que no se corresponden con su aspecto original, dificultan la datación y el estudio directo.

Al comienzo del Protectorado Español en Marruecos (1912-1956) la medina de Tetuán con sus murallas y puertas, fueron percibidas, desde un primer momento, como parte fundamental de la ciudad tradicional que se debía conservar. En la Cartoteca del Servicio Geográfico del Ejército localizamos los primeros levantamientos que se realizaron a escala 1:100 de las puertas de la medina. Están firmados por Francisco Gómez Jordana; Alejo Corso y Eduardo Álvarez en 1888 .

El objeto del presente estudio es dar a conocer los planos de la Bab at-Tut o Puerta Tánger, que se realizaron durante la ocupación española; facilitar una descripción a partir de la documentación gráfica que se conserva y, por último, realizar un levantamiento planimétrico actualizado. Con todos estos elementos se podrá establecer un criterio restaurador que facilite en el futuro una correcta recuperación del patrimonio defensivo de la ciudad de Tetuán. 


\section{Breve descripción de la medina de Tetuán}

La ciudad de Tetuán se encuentra situada en el llamado trapecio norte de Marruecos. En el territorio de Anyera, a pocos kilómetros de la costa mediterránea y a escasa distancia del Estrecho de Gibraltar, sobre unas terrazas del río Martin, en la falda del monte Dersa. Sus características geográficas han tenido como consecuencia el que fuese zona de paso y de penetraciones (al ser el único camino terrestre para llegar a Ceuta), y de asentamientos, en especial de los numerosos granadinos que, entre otras cosas, encontraron en esta tierra tanta semejanza con la que acababan de abandonar (Gozalbes 1988). Su accidentada orografía, a su vez, ha servido al tetuaní para permanecer un tanto independizado del resto del país, llegando incluso, en ciertos momentos, al enfrentamiento y al no reconocimiento de la autoridad establecida.

La medina amurallada de Tetuán, es de trazo irregular con una forma cercana a la triangular. Está dividida en cinco grandes barrios, cuyos nombres son: al-Blad, al-Ayum, Trancatz (la conjunción de los dos en la zona Este, también es conocida como Haumat Tala'a), es-Suica (que algunos dividen en Rbat es-Sefli y Sidi Meshba) y Mellah.

Cada una de sus calles principales parte de una de sus siete puertas ${ }^{1}$, actualmente abiertas, que se sitúan en su muralla. Estas calles, aunque son sensiblemente radiales, no confluyen en un mismo lugar. En la parte más alta del recinto murado y tangente a él, de acuerdo con la tradicional arquitectura militar, se encuentra la Alcazaba.

\section{Cartografía de Tetuán}

La primera referencia cartográfica, que se conserva de la ciudad de Tetuán, con rigor descriptivo para reconocer el trazado de la muralla y las puertas del recinto, es de $1860^{2}$ (Fig. 1). En su recorrido se observan algunas irregularidades o imprecisiones, pero la muralla está representada con corrección y las puertas están referenciadas por sus nombres españoles (Ruiz de Cuevas, 1951).
Otra fecha importante para la historia cartográfica de Tetuán, es la de 1882. En este año la Comisión de Estado Mayor envía a Marruecos una misión de reconocimiento e información territorial con el encargo de realizar un levantamiento de las principales ciudades marroquíes. Los motivos de estos levantamientos son muy diversos, pero sin duda no faltaban las aspiraciones coloniales y el deseo de posicionarse más allá de la franja de territorio que le fue asignada en el convenio hispano-francés de 1904 (Urteaga, Nadal, Muro, 2003, p. 17).

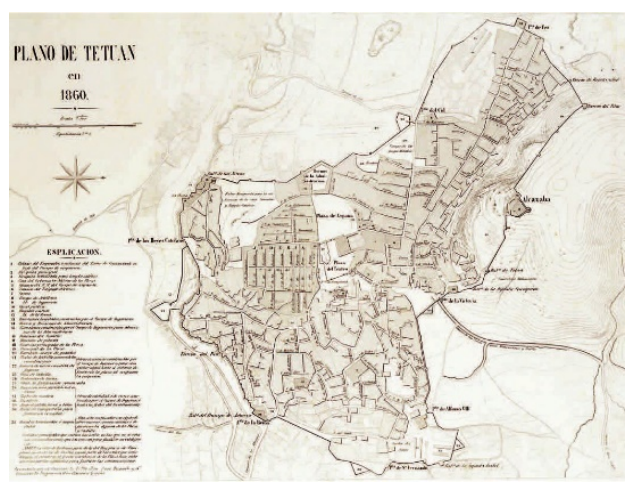

Fig. 1. Plano de Tetuán en 1860 . Hoja $\mathrm{n}^{\circ} 8$. Atlas histórico y topográfico de la guerra de África. 1859-1860 (Archivo del Servicio Geográfico del Ejército, Madrid).

De esta operación cartográfica son los trabajos realizados sobre la ciudad de Tetuán, realizados a escala 1:2500. Muestran una cuidadosa representación del núcleo urbano y de la zona de huertas que rodea la ciudad (Fig. 2). Sin embargo, se podría decir que hasta 1888 no se levantaron los planos de la ciudad, pues es cuando tanto las murallas, como las puertas de la medina tuvieron verdadero protagonismo cartográfico y por tanto patrimonial, ya que sirvieron como estaciones geodésicas y elementos de referencia planimétrica. Se comprueba además que estos elementos arquitectónicos, fueron percibidos desde un primer momento, como parte fundamental de la ciudad tradicional que se debía conservar. Y de ahí la preocupación que se tuvo por obtener una adecuada representación gráfica de los monumentos incluso en los momentos bélicos como fue la Guerra de Tetuán de 1859. 


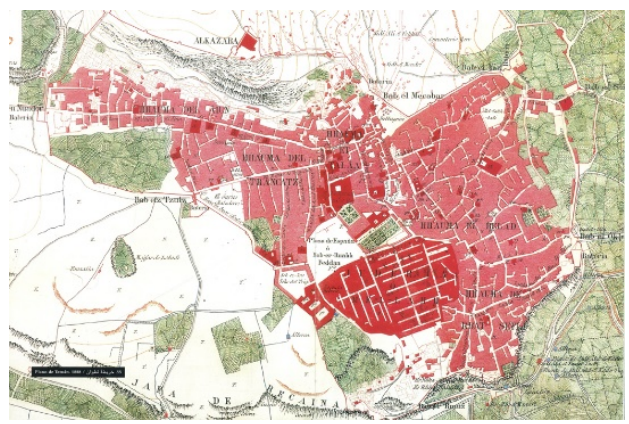

Fig. 2. Croquis de Tetuán y sus Alrededores en 1888 (Archivo del Servicio Geográfico del Ejército, Madrid).

En la Cartoteca del Servicio Geográfico del Ejército (Madrid) encontramos los primeros levantamientos que se realizaron a escala 1:100 de las puertas de la medina (En concreto de la Bab Nuader, Bab Oqla y Bab Tut). Están firmados por Francisco Gómez Jordana; Alejo Corso y Eduardo Álvarez en $1888 .^{3}$

\section{Bab Tut}

$\mathrm{La} \mathrm{Bab}$ at-Tut, es un conjunto de dos puertas, ambas de acceso directo. Conocida como la "Puerta de Tánger" por dar acceso al camino que conducía a esta ciudad; o también llamada Puerta de las Moreras, por la abundancia de estos árboles, que extendía sus ramas en las huertas de este sector (Joly, 1905, p. 280).

Durante la ocupación española de 1860 se la llamó Puerta del Cid (Ruiz de Cuevas, 1951, p. 37). Está situada al oeste del recinto murado, y fue reconstruida y trasladada al norte de su emplazamiento original cuando se derribaron las murallas en la etapa del Protectorado español. ${ }^{4}$

\subsection{Situación}

Está situada al sur-oeste de la muralla de la ciudad, y es la entrada del barrio del Trankat, al final de la calle de igual nombre y la de Dar alBomba (Fig. 3). Además de las huertas mencionadas, en el exterior estaban los barrios de Ain- Al Mazuak, el de Sidi Idris, el de Ain AlJabbaz y el de Kanatir. Sobre alguno de ellos se construyó el "Ensanche" (Malo, Domínguez, 1994).

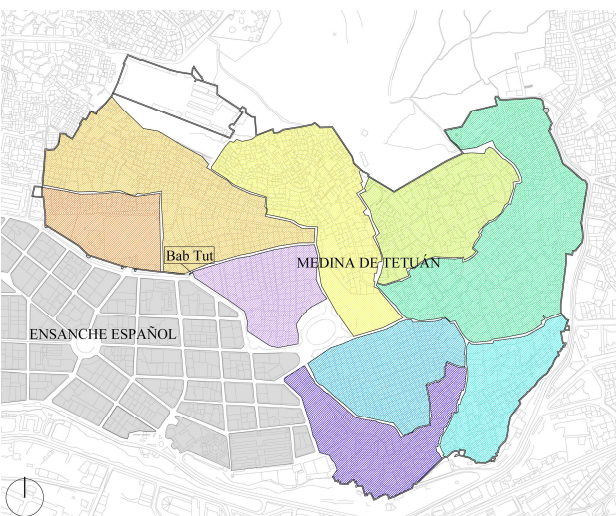

Fig. 3. Ubicación de Bab Tut en la medina (Autores).

\subsection{Datación}

Existen datos contradictorios en cuanto a la realización de esta puerta. Algunos historiadores indican que la Puerta primitiva fue construida a finales del Siglo XVI o principios del XVII, por los andalusíes rebelados en la Alpujarra granadina y que al ser expulsados se aposentaron en el Rabal tetuaní que luego llevó el nombre de Trankat, al que da acceso dicha puerta. Otros relatan que la Puerta fue construida antes del siglo XVIII, ya que, a mitad de ese siglo (1168 H.), el Hach Muhamad ben Umar Lukas mandó construir tanto el Arco como la fuente anexa. ${ }^{5}$

En 1808 el Sultán Muley Soleyman al mandar cerrar este sector del perímetro amurallado, hizo construir la puerta anexa, que era casi perpendicular a la anterior (alzado sur representado en alzado en la Fig. 4).

En 1915, el arquitecto Carlos Óvilo Castelo urbaniza la parte de la muralla frente al cuartel de artillería y la Puerta de Tánger. Hasta 1927 sólo constaba del arco central de ingreso. En ese año se le abrieron los dos arcos laterales, diferenciando y facilitando así el tráfico rodado del peatonal (Fig. 6). Estos portillos son obra del arquitecto Lescura, habiendo sido antes habitaciones del cuerpo de guardia. Para modificar estos portillos Manuel Latorre Pastor reclamó la opinión de Leopoldo Torres Balbás.

Esta puerta se elevaba como un puente simbólico entre la medina y el Ensanche, pero, más tarde, en los años 50, y a consecuencia de que la 
circulación automovilística sufría un penosísimo embotellamiento, ocasionando accidentes, se demolió "por tratarse de arquitectura decadente y de escaso valor artístico" para permitir el paso de los vehículos, especialmente el trolebús, quedando la Puerta tal como debió ser concebida primitivamente.

Este acceso tenía dos partes claramente diferenciadas, como eran la puerta propiamente dicha, que daba entrada al barrio del Trankat, con una fuente monumental anexa y otro acceso añadido casi perpendicular al anterior.

De ambos accesos partían sendas murallas, que formaban una especie de pasillo, en el que había instalados una serie de barracas, utilizadas como bakalitos, adheridos a los dos muros. Actualmente sólo persisten los dos primeros elementos, ya que la puerta añadida fue derribada a finales de los años 50.

La Puerta primitiva está formada por un cuerpo paralelepipédico, en el que la altura predomina sobre la anchura. Su planta es rectangular y dispone de dos vanos, uno interior y otro exterior.

\subsection{Descripción}

La fachada exterior está enmarcada, a manera de un doble alfiz que llega hasta el suelo, por dos

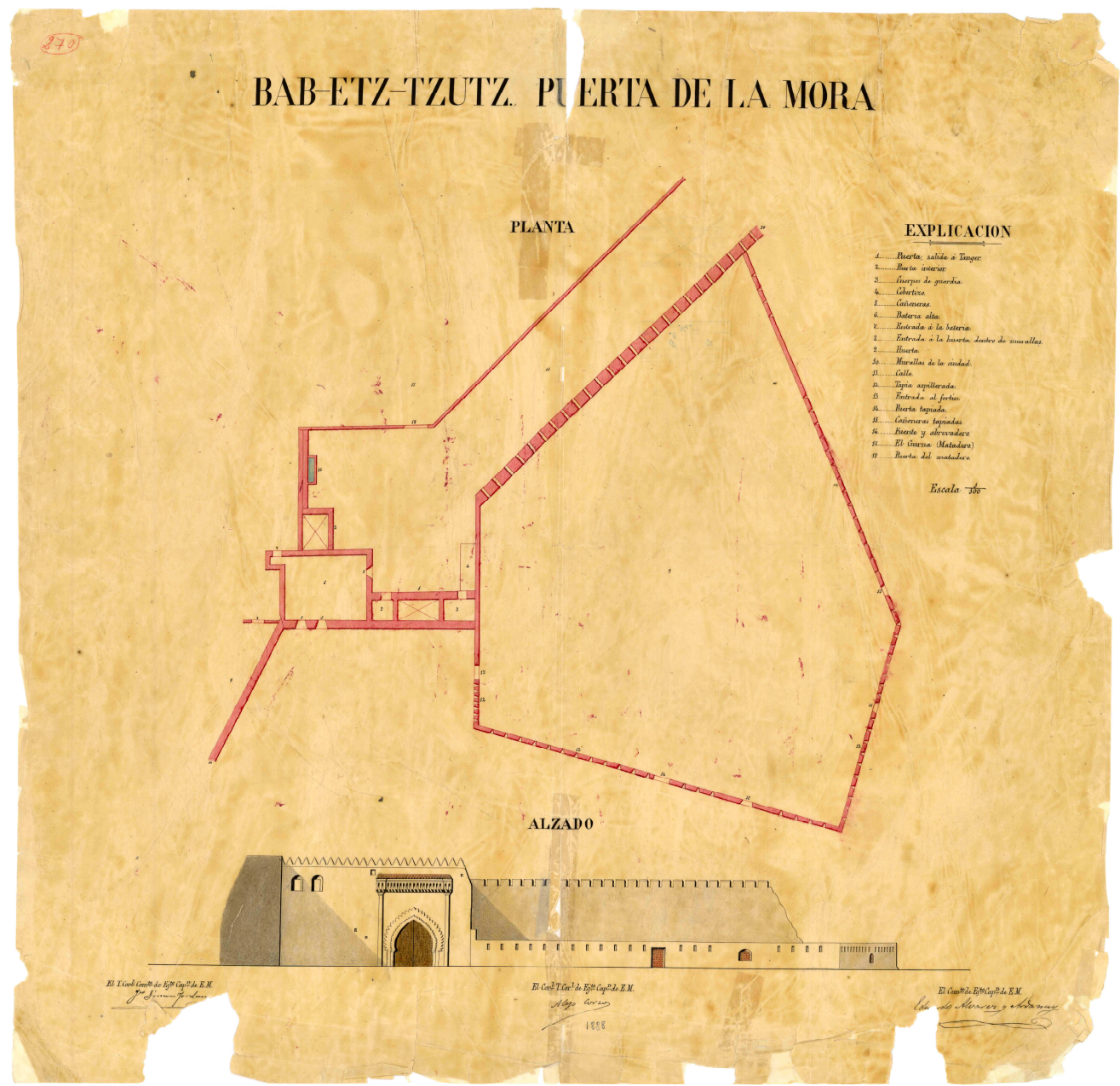

Fig. 4. Planta y Alzado de Bab Tut, 1888 (Archivo del Servicio Geográfico del Ejército, Madrid). ${ }^{6}$ 
rehundidos terminados en canto vivo, a excepción de la parte superior del primero, que es una moldura con esgucio y regleta. Sobre este enmarcamiento hay una cenefa de azulejo esgrafiado, que forma un friso de 4,20 x 0,22 m. La grafía se debe al calígrafo Muley 'Aysà-l-Yaziri. $\mathrm{Su}$ traducción, según Fernando Valderrama (Valderrama Martínez, 1954, 1975, p. 30), dice: "Me refugio en Dios contra Satán el lapidado. En el nombre de Dios Clemente y Misericordioso. Mi éxito sólo depende de Dios. Él me sostiene y a Él volveré. Hágase la voluntad de Dios. Sólo Él posee la fuerza." (Figs. 5 y 6).

El arco del vano es de herradura apuntado, con unos simples arranques de quiebro horizontal. Concéntrico a él, otro arco angrelado lo cobija. Sus lóbulos son pequeños arquitos de medio punto. Antiguamente, sobre el friso de cerámica, había un ventanuco central y cuatro aspilleras, colocadas simétricamente respecto al ventanuco.

El muro acaba en liso. El lateral derecho se prolonga hasta una cierta altura acabando en un plano inclinado con una fuerte pendiente hacia el interior. El lateral izquierdo estaba, antiguamente adherido a una especie de torre situada en la intersección con la Puerta, sin embargo, al desaparecer este cuerpo, posiblemente en el año 1927, se terminó ese lateral de la misma forma que el opuesto.

La fachada interior no presenta ninguna característica especial, salvo el arco del vano que es exactamente igual que el exterior.

Existía una puerta añadida con forma paralelepipédica, en la que la longitud y la altura prevalecían sobre su anchura, y que forma un ángulo de $115^{\circ}$ con la Puerta. Constaba de un grueso muro de mampostería coronado por las típicas almenas dentadas. La planta era oblonga en la que se apreciaba un vano de acceso central y dos portillos, de la mitad de la luz libre, simétricamente colocados a ambos lados de la puerta central. En la parte superior de la construcción se encontraba una especie de habitación que contaba con dos bocas de fuego.

En la fachada interior sólo se distinguían los tres huecos de paso, compuestos por arcos de herradura ligeramente apuntados, sin ningún tipo de decoración, a excepción de un delgado filete que a todo lo largo de la fachada parecía marcar la altura del suelo interior de la segunda planta. Además, y rematando la fachada había unas pequeñas almenas.

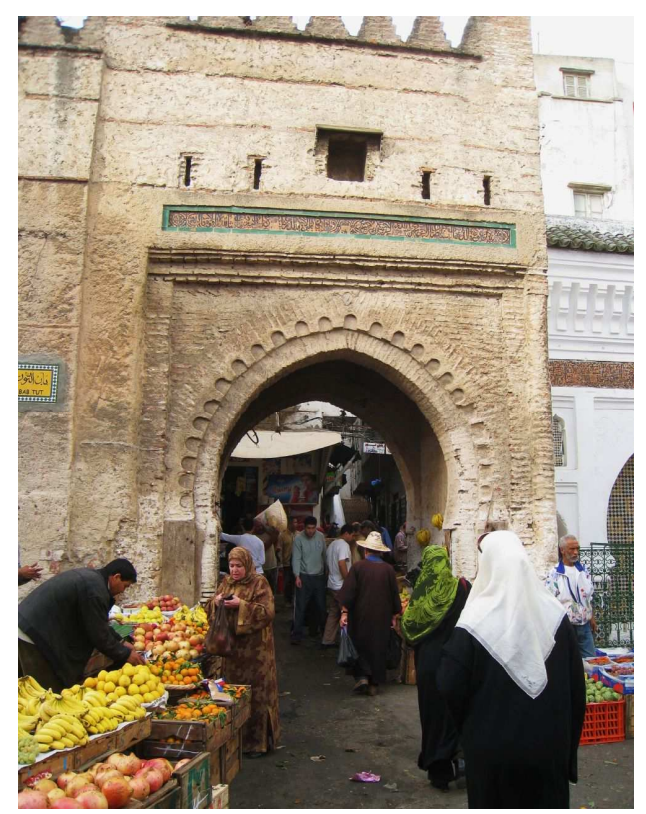

Fig. 5. Bab Tut en la actualidad (Autores).

La fachada exterior era asimétrica, ya que la puerta y los dos portillos laterales no estaban situados en el eje de simetría del conjunto. El vano central, estaba encuadrado por una portada típica, característica por lo que se ha llamado barroco tetuaní. Se encontraba enmarcado por dos pilastras, o antas, achaflanadas, que acababan con sección rectangular por medio de pequeñas pechinas o triángulos esféricos, que, con una moldura en esgucio, componían un modesto capitel. Esta moldura se prolongaba entre ambas antas. Encima había un ancho friso mensulado, compuesto por cartelas de dobles esgucios con dobles regletas, unidas por arcos de medio punto. La superponen dos fajas acodaladas, siendo la superior más ancha que la inferior y es sobre la que se apoyan las bocatejas del tejaroz, del que sobresale una parte del macizo del muro.

El arco central era de herradura ligeramente apuntado, cuyos arranques eran unos esgucios y regleta. Concéntricamente a él, presentaba un 
doble festón de arquitos, o de arcos lobulados, a la manera de arquivolta rehundida. El interior poseía dos tipos de lóbulos, el más ancho formado por un simple arquito ciego de medio punto y, el otro, de la mitad de la luz del anterior, era un arco apuntado. En el del exterior todos los lóbulos son iguales y lo componían arquitos mixtilíneos. Los arranques de ambos eran semejantes a los correspondientes al arco principal, pero de menor altura.
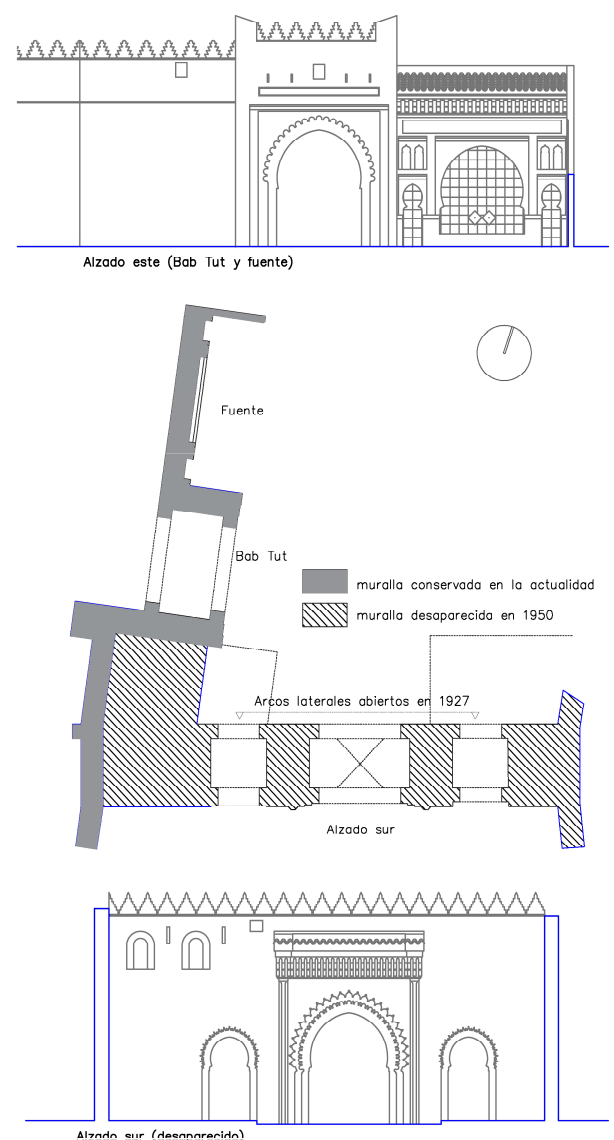

Fig. 6. Bab Tut en la actualidad (alzado); planta (actual y muralla desaparecida); alzado sur (desaparecido).

Los arcos de los portillos, eran iguales entre sí y lo formaban unos arcos semejantes al central, pero de menor radio. Estaban enmarcados por otro lobulado, concéntrico. Estos lóbulos eran dos tipos, uno de arco de medio punto de pequeñísimas dimensiones alternado con otros mixtilíneos, algo mayores. El conjunto da la sensación de un arco angrelado convexo.
La asimetría comentada venía dada por dos viejas troneras, que se abrían al exterior, a nivel del entablamento del arco central. Se encontraban en desequilibrio con la otra parte, ya que estaban situadas en la zona más meridional de la fachada de la construcción, es decir, a la derecha cuando se mira la muralla desde fuera. Se trataba de dos arcos de medio punto, abocinados y por los que asomaban las bocas de los dos cañones pedreros. A la derecha, de cada una de ellas, se abrían dos delgadas aspilleras. También se podía distinguir un pequeño ventanuco cuadrado.

La techumbre del recinto central estaba formada por una bóveda de cañón y su cubierta era plana. Un coronamiento de almenas o merlones dentados, sobre un largo y delgado filete, completa el carácter de esta fachada.

\subsection{Otros elementos}

- El Torreón. En la conjunción de ambos accesos existía, según fotografías antiguas, un saliente en ángulo, que semejaba a un torreón, que posteriormente desapareció. Su altura era semejante a la puerta actualmente existente y estaba rematado por almenas del mismo tipo que el resto. En sus fachadas abrían, además de un ventanuco unos huecos pequeños.

- La fuente. Está retranqueada respecto al arco de paso y presenta una superficie semejante a una fachada. En su parte inferior está dividida en tres fajas, una ancha central que contiene a la fuente propiamente dicha y otras dos laterales mucho más estrechas. La central es un simple arco ciego de herradura apuntado con arranques en esgucio y listel cobijado bajo un alfiz terminado en canto vivo a excepción de la parte superior que dispone de una moldura en esgucio y regleta. La parte inferior es el vaso de la fuente.

Las zonas laterales están, a su vez, divididas en dos partes, la inferior es un arco ciego, aunque profundo, de herradura apuntado, remarcado por un alfiz, que, al igual que el del arco central, es de canto vivo y acaba con el mismo tipo de moldura en su parte superior. Sobre estos hay unos rehundidos, rectangulares, que albergan, cada uno a dos arquitos ciegos pareados y con una línea de intrados con un trazado de arco conopial tumido o de herradura. La parte superior del 
rehundido acaba con la misma moldura que los alfices.

Encima corre un friso de azulejos esgrafiados, semejante al existente en el Arco, de unas dimensiones de 5,60 x 0,44 m, cuya leyenda, del mismo calígrafo que la anterior, traducida también por Valderrama (Valderrama Martínez, 1975, p. 31) dice:

"Detente, visitante, en estado de virtud y así mismo el que pasa y el que llega / Considera con atención mi obra de arte / Su estructura es la belleza de la delicada perfección / Me ha construido el virtuoso y amado, el que excede en generosidad a Hatim / Lukas, heredero de la excelencia, de la nobleza y de las virtudes; / Mamad, hijo del recto imán de todos los sabios. / La fecha de mi construcción está en el verso de un poeta ${ }^{7} /$ Perdura bellamente la perfección de mi figura gracias al gobernador. / El manantial de mi fuente está en la generosidad de la pureza de las nubes. / Te daré a beber agua dulce de su kaular con sello de almizcle / Bebe agua sana, saludable, augurio de salud eterna. / Pide a Dios, ante ella, para mi dueño, victoria y trofeos."

Encima corre un friso mensulado compuesto por cartelas de dobles esgucios y dobles regletas unidas por dinteles, sobre el que se extiende una moldura, también de esgucio y regleta que se remata con las bocatejas del tejaroz.

Antiguamente no estaba revestida, pero en la actualidad presenta en todos los fondos de los tres arcos y en los machones que forman los estribos y hasta la línea de impostas, alicatado de estilo tetuaní.

\section{Conclusiones}

Con este análisis hemos obtenido una descripción morfológica de la Bab Tut. Es una construcción modesta, sin grandes pretensiones estilísticas, pero sí funcionales. Del estudio de este elemento se desprende que no se incorpora en su configuración, ningún elemento estilístico nuevo. Se reutilizan los ya conocidos a través de alAndalus. Quizá por las continuas refundaciones de la ciudad de Tetuán y esa vocación de ciudad de paso hayan impregnado el modo de ser estilístico hispano-magrebí. ${ }^{8}$

Como detalle de elemento compositivo se puede destacar que la moldura empleada por excelencia es la nacela, que, combinada con regleta, aparece como arranques de arcos, como límite de paños, en los perfiles de las cornisas, arquitrabes, y en los modillones.

\section{Notas}

${ }^{1}$ Estas son: Bab Nuader, Bab Tut, Bab Remuz, Bab Oqla, Bab Saida, Bab Jiaf y Bab Mqabar.

${ }^{2}$ Está catalogado con el número 35 entre los planos de Tetuán, considerados como históricos en el Servicio Geográfico del Ejército (ASGE).

${ }^{3}$ Son planos poco precisos de escala. En la planta se altera la distribución de huecos mezclando planta baja y planta primera. Se representa el Alzado Sur, perpendicular a Bab Tut. Este añadido está construido en 1808. En 1927 se le añadirán dos arcos laterales (ver figura 6 dibujo inferior).

${ }^{4}$ Sobre el derribo de la muralla que separa el Ensanche de la calle Tánger, véase África Española, 30 de marzo de 1916, nº 34, pp. 328.

${ }^{5}$ Dato tomado de la leyenda caligrafiada en los azulejos del friso. Traducido por Fernando Valderrama (1975, p. 31).

${ }^{6}$ Planta y Alzado de Bab Tut o Puerta De La Mora (1888). E: 1/100. Eduardo Alvarez y Ardanuy; Alejo Corso; F. Gómez Jordana. Descripción física: 1 plano: ms., col., montado sobre papel; $72 \times 74 \mathrm{~cm}$, pleg. en $38 \times 73 \mathrm{~cm}$. Manuscrito firmado y rubricado, a plumilla en tinta negra y carmín iluminado a la acuarela en siena. Archivo del Servicio Geográfico del Ejército. (Madrid). Ref.: Ar.Q-T.9-C.3-145.

${ }^{7}$ Sumados los valores de las letras que forman el verso siguiente dan un total de 1168 , correspondiente al año de la construcción de la fuente.

${ }^{8}$ Sobre el estilo hispano-magrebí se puede consultar (González Alcantud, 2008) los diferentes artículos de la obra de González Alcantud. 


\section{Bibliography}

Gozalbes, G. (1988). Al-Mandari, el granadino fundador de Tetuán, Obra Cultural de la Caja de Ahorros de Granada Ed., Granada.

González Alcantud, J.A., coord. (2008). La invención del estilo Hispano-Magrebí, Anthropos, Barcelona.

Joly, M.A. (1905). “Tetouan”, Archives marocaines: publication de la Mission Scientifique du Maroc, pp. 199-345.

Malo de Molina, J.; Domínguez, F. (1994). Tetuán: El Ensanche: guía de arquitectura, 1913-1956, Consejería de Obras Públicas y Transportes de la Junta de Andalucía, Sevilla.

Ruiz de Cuevas, T. (1951). Apuntes para la historia de Tetuán, Imnasa, Tetuán, p. 65.

Urteaga, L; Nadal, F.; Muro J.I. (2003). "Imperialismo y cartografía: la organización de la comisión española de Estado Mayor en Marruecos (1881-1882)", Geo crítica/Scripta Nova, VII, 142, pp. 13-33.

Valderrama, F. (1954). "Dos inscripciones árabes en Bab t-Tut de Tetuán”, Hesperis-Tamuda, 41, pp. 459-462.

Valderrama, F. (1975). Inscripciones árabes de Tetuán, Instituto Hispano-Arabe de Cultura, Madrid. 